\title{
Reflujo faringolaríngeo: Revisión de los nuevos métodos diagnósticos
}

\section{Laryngopharyngeal reflux: A review of new diagnostic methods}

\author{
Christian Olavarría L' ${ }^{1}$, Pedro Cortez V'1 .
}

\section{RESUMEN}

El reflujo faringo laríngeo (RFL), es una causa común de morbilidad del tracto aerodigestivo superior. A pesar de su alta prevalencia, aún existe controversia respecto de la precisión diagnóstica de esta enfermedad, así como de su fisiopatología y manejo. Los test diagnósticos actuales aún presentan limitaciones significativas, de ahí que surja el interés por encontrar métodos rápidos y eficientes que mejoren su pesquisa. El presente trabajo tiene por objeto la revisión de la literatura actual en cuanto a los métodos disponibles para su diagnóstico.

Palabras clave: Laringitis, reflujo faringolaríngeo, pH-metría, pepsina en saliva.

\begin{abstract}
The laryngopharyngeal reflux is a common cause of morbidity of the upper aerodigestive tract. Despite its high prevalence, there is still controversy regarding the diagnostic accuracy of this disease and its pathophysiology and management. The current diagnostic test still have significant limitations, hence the interest arises to find fast and efficient methods to improve their research. This paper aims to review the current literature regarding available methods for diagnosis.

Key words: Laryngitis, laryngopharyngeal reflux, pH monitoring, Salivary pepsin test.

\section{INTRODUCCIÓN}

gología. Se estima que la prevalencia del RFL de $10 \%-30 \%$ de la población occidental ${ }^{1}$. En el año 2006, el Consenso de Montreal definió la existencia de síndromes extraesofágicos establecidos que se manifiestan con tos, laringitis, asma y erosiones dentales ${ }^{2}$.
\end{abstract}

El RFL, que corresponde a una variante de la enfermedad por reflujo gastroesofágico (ERGE), es una de las condiciones más comúnmente diagnosticadas en la práctica de la otorrinolarin-

\footnotetext{
${ }^{1}$ Servicio de Otorrinolaringología, Hospital Clínico Universidad de Chile. 
El interés por RFL ha crecido sustancialmente en las últimas dos décadas. A pesar de la gran cantidad de estudios existentes al respecto, aún persiste la controversia en cuanto a la precisión diagnóstica de la enfermedad, su fisiopatología y la eficacia de tratamiento. Los test diagnósticos actuales aún presentan limitaciones significativas, de ahí que surja el interés por encontrar métodos rápidos y eficientes que mejoren la aproximación diagnóstica. El rol de la endoscopía digestiva alta (EDA) en la ERGE es muy limitado, pues la prevalencia de hallazgos endoscópicos en pacientes con síntomas de RGE no supera el $30 \%{ }^{3}$. La medición del reflujo empleando la pHmetría ambulatoria y/0 el monitoreo de la impedancia como test diagnósticos para ERGE tienen sensibilidad (77\%-100\%) y especificidad (85\%-100\%) bastante aceptables en pacientes con signos de esofagitis endoscópica ${ }^{4}$. Sin embargo la sensibilidad es mucho menor en los casos de EDA normal. Entonces, tanto la EDA como los test de monitoreo de reflujo ambulatorios son subóptimos para el diagnóstico de ERGE además de ser costosos e invasivos. Esto se magnifica en el diagnóstico de enfermedad extraesofágica por reflujo, por la baja sensibilidad de la EDA, del monitoreo de $\mathrm{pH}$ y los hallazgos inespecíficos de la nasofibrolaringoscopía (NFL).

En los últimos años ha aparecido en el mercado un dispositivo de detección rápida de pepsina en saliva, que se plantea como un método objetivo para diagnosticar reflujo (PEPTEST ${ }^{\mathrm{TM}}$ ).

El motivo del presente artículo es revisar la evidencia actual respecto de los nuevos métodos diagnósticos en el RFL.

\section{FISIOPATOLOGÍA}

Se cree que el RFL es causado por el flujo retrógrado de contenido gástrico (particularmente de ácido y pepsina) que afecta la mucosa faríngea y laríngea por contacto directo o por algún mecanismo secundario. Esto último pudiese estar dado por la irritación ácida del esófago distal, que causa tos y carraspeo crónico mediado por el nervio vago y así actuar como un factor mecánico de daño larínge $0^{5}$. Además, se ha demostrado que el reflujo de sales biliares también pueden causar irritación de la mucosa laríngea ${ }^{6,7}$. En el año 2010,
Habesoglu ${ }^{8}$ y cols demostraron en un modelo animal que la exposición de la mucosa laríngea al ácido y pepsina provoca edema de la lámina propia, hiperplasia de la glándulas submucosas y atrofia muscular. Erickson y cols ${ }^{9}$ describieron que menos de tres episodios por semana de reflujo ácido sobre la mucosa laríngea son suficientes para provocar alteraciones histológicas en las cuerdas vocales, a diferencia de la mucosa de esófago distal que es capaz de tolerar hasta 50 episodios de reflujo por día y que se considera fisiológico en esta zona.

Es probable que los pacientes con respuesta insuficiente a la supresión ácida puedan tener la participación de los otros componentes del reflujo gástrico, tales como pepsina y los ácidos biliares. La pepsina es la principal enzima proteolítica producida en el estómago. Se ha demostrado que la pepsina está presente en el epitelio laríngeo de los pacientes con enfermedad de la laringe atribuida al reflujo ${ }^{10}$. Incluso, se ha determinado una asociación significativa entre la presencia de pepsina en epitelio laríngeo en los pacientes con RFL y la depleción de dos proteínas que otorgan protección a la mucosa de la laringe. Una es la anhidrasa carbónica isoenzima III (CAIII) y la otra es la proteína de estrés epitelial de tejido escamoso (Sep70) ${ }^{11}$. Se conoce que la actividad máxima de la pepsina se logra en un medio ácido ( $\mathrm{pH}<2)$ y que ésta se inactiva con $\mathrm{pH}>6,5$, siendo el $\mathrm{pH}$ promedio normal en la faringolaringe de 6,8. Mediante un modelo animal in vitro se logró determinar que la pepsina que se aisla en la mucosa laríngea luego de un evento de reflujo, puede conservarse inactiva hasta por al menos $24 \mathrm{hrs}$ (a un pH no superior a 8 a $37^{\circ} \mathrm{C}$ de temperatura), siendo capaz de reactivar hasta el $80 \%$ de su capacidad enzimática cuando el pH en su entorno se reacidifica ${ }^{12}$.

\section{DIAGNÓSTICO}

\section{Prueba terapéutica}

Considerando que el RGE/RFL es un fenómeno que ocurre en el $65 \%-75 \%$ de personas sanas ${ }^{13}$, y a pesar de los múltiples estudios publicados, aún no existe un examen que se considere como un verdadero gold standard para la detección de RFL patológico ${ }^{13-15}$. Hasta ahora, el diagnóstico 
de reflujo extraesofágico se basa en la sospecha clínica sumado ya sea, a la respuesta a inhibidores de la bomba de protones (IBPs) 0 a estudios de $\mathrm{pH}$ supraesofágico positivos ${ }^{16}$. Los que proponen la "prueba terapéutica" con IBPs se basan en la inexistencia de criterios y métodos bien definidos para detectar la presencia de reflujo supraesofágico así como la baja correlación entre eventos de reflujo y la respuesta a IBPs $^{17,18}$. La respuesta a esta "prueba terapéutica" con IBPs, aún carece de estudios bien controlados serios como para permitir sacar conclusiones razonables ${ }^{15}$.

\section{pH-metría}

El grupo de Ayazi y cols ${ }^{19}$ plantean un nuevo paradigma para el diagnóstico de RFL basado en la pérdida de la alcalinización en el esófago cervical. Ellos observaron en sus pacientes con RFL, una incapacidad para mantener un $\mathrm{pH}$ neutro en esta zona, basado en una mayor exposición a un $\mathrm{pH}<4$ y una menor exposición a un $\mathrm{pH} \geq 7$, en comparación con los pacientes sanos. Estos autores sugieren que la sensibilidad para detectar el reflujo anormal y su relación causal con la síntomas faringolaríngeos se incrementa al considerar tanto el mayor porcentaje de tiempo en que se registra un $\mathrm{pH}<4$, como el menor porcentaje de tiempo con $\mathrm{pH} \geq 7$. Ellos consideran que la pérdida de un medio alcalino $(\mathrm{pH} \geq 7)$ en el esófago cervical es un indicador de la disminución de su capacidad para proteger los tejidos de daños, en contraste con el tiempo de $\mathrm{pH}<4$, que hasta ahora ha sido aceptado como causantes de los cambios patológicos asociados con la sintomatología de RFL ${ }^{19}$. Por el momento, la pH-metría de 24 hrs es el examen más relevante para cuantificar el reflujo esofágico en pacientes con síntomas de $\mathrm{RGE}^{20}$. Originalmente, se usaba un catéter con sensor único ubicado $5 \mathrm{~cm}$ sobre el esfínter esofágico inferior (EEI). Posteriormente se agregó un segundo sensor a esta sonda a nivel de hipofaringe, llegando a ser considerado por mucho tiempo como gold standard para el diagnóstico de RFL. Sin embargo aún no hay consenso respecto de la ubicación de los sensores, la cantidad de sensores requeridos y la interpretación de sus resultados. En 2009 la compañía Restech desarrolló una prueba que mide el pH tanto "aerolizado" como del entorno líquido de la orofaringe, siendo una prueba menos invasiva y más tolerable para el paciente con buen resultado clínico ${ }^{21}$. Esta compañía y grupo de estudio plantean incluso que el criterio tradicional de $\mathrm{pH}<4$ como indicador de RFL no es utilizable en orofaringe. Sugieren que un evento significativo sería aquel que sobrepasa las 3,5 "desviaciones standard" del pH de base ${ }^{22}$.

Sato y $\operatorname{cols}^{23}$ describieron su experiencia utilizando un sistema de monitoreo de $\mathrm{pH}$-metría de 24 hrs usando 4 sensores. El proximal ubicado en hipofaringe, el segundo en tercio medio esofágico, el tercero a unos pocos $\mathrm{cm}$ sobre EEI (no a $5 \mathrm{~cm}$ como las demás) y el distal ubicado en el estómago. Presentaría la ventaja de poder registrar en forma simultánea el pH en todas estas localizaciones pudiendo cotejar la relación entre ellas. El problema, es que no es posible asimilar sus resultados con las otras pruebas que presentan el sensor esofágico a $5 \mathrm{~cm}$ del EEI. En sus estudios concluyen que tanto los valores de $\mathrm{pH}$ menor a 4 como menor a 5 son significativos e indicadores de RFL.

\section{Impedanciometría intraluminal}

La impedanciometría intraluminal multicanal asociado al monitoreo de $\mathrm{pH}(\mathrm{IIM} / \mathrm{ph})$ es una técnica relativamente nueva que es considerada como el gold standard actual en la detección de $\mathrm{RFL}^{20}$. Esta prueba es capaz de detectar episodios de reflujo en su forma líquida, como también en forma de gas o mixtas. Incluso detecta tanto el reflujo ácido como no ácido. La IIM se basa en los cambios en la resistencia eléctrica al flujo de una corriente eléctrica entre dos electrodos colocados en una sonda, cuando un bolo líquido, semisólido 0 gaseoso pasa entre ellos ${ }^{24}$. En general, se utilizan varios catéteres "adecuados según edad" para los estudios de impedanciometría. Existen 7 sensores de impedancia colocados a lo largo de cada catéter, que dan como resultado 6 canales de impedancia correspondientes. El electrodo de $\mathrm{pH}$ se coloca en el centro del espacio del sensor de impedancia más distante (catéteres infantiles y pediátricos) 0 en el espacio del sensor de impedancia que se encuentra inmediatamente más próximo a él (catéteres para adultos). Existen también catéteres con 2 puntos de medición de pH. Un episodio de reflujo por impedancia se define como una caída en 
la impedancia intraluminal de $\leq 50 \%$ desde el valor basal que progresa de modo retrógrado a través de 2 o más de los canales más distantes. Un episodio se considera ácido cuando el pH esofágico disminuye y permanece en $<4$ durante al menos 5 segundos. Un episodio se considera no ácido cuando el pH aumenta, permanece sin cambios o disminuye en 1 unidad de $\mathrm{pH}$ mientras permanece $\geq 4$.

Lee y cols ${ }^{25}$ documentaron un aumento sustancial en el diagnóstico de reflujo usando esta prueba respecto del monitoreo de $\mathrm{pH}$ clásico. Loots y cols ${ }^{26}$ reportaron un aumento en el rendimiento del diagnóstico al combinar el IIM con la pH-metría en niños y lactantes con reflujo confirmado.

\section{Cuestionario e índices}

El índice de síntomas de reflujo (RSI - Reflux Symptom Index) ${ }^{27}$ [Tabla 1], fue diseñado para medir la severidad de síntomas laríngeos, y el score de hallazgos endoscópicos de reflujo (RFS - Reflux Finding Score $)^{28}$ [Tabla 2], se utiliza para graduar los hallazgos a la fibroscopía laríngea. Ambas herramientas han sido utilizadas desde hace más de una década, ayudando en la aproximacíon clínica al diagnóstico de RFL. En los últimos años, Anderson y cols ${ }^{29}$, desarrollaron un cuestionario de síntomas de reflujo faríngeo, autoaplicado, que presenta una fuerte correlación con el RSI. La ventaja principal

Tabla 1. Indice de síntomas de reflujo (RSI)

\begin{tabular}{|c|c|c|c|c|c|c|}
\hline \multicolumn{7}{|l|}{ Durante el mes pasado, ¿cómo lo afectaron los siguientes problemas? } \\
\hline 1. Disfonía u otro problema con su voz & 0 & 1 & 2 & 3 & 4 & 5 \\
\hline 2. Carraspera & 0 & 1 & 2 & 3 & 4 & 5 \\
\hline 3. Presencia de moco excesivo en su garganta o goteo retronasal & 0 & 1 & 2 & 3 & 4 & 5 \\
\hline 4. Dificultad para deglutir alimentos líquidos o pastillas & 0 & 1 & 2 & 3 & 4 & 5 \\
\hline 5. Tos después de comer 0 acostarse & 0 & 1 & 2 & 3 & 4 & 5 \\
\hline 6. Sensación de ahogo o atrancamiento & 0 & 1 & 2 & 3 & 4 & 5 \\
\hline 7. Tos ocasional o en accesos & 0 & 1 & 2 & 3 & 4 & 5 \\
\hline 8. Sensación de taco o una aguja en su garganta & 0 & 1 & 2 & 3 & 4 & 5 \\
\hline 9. Quemadura retroesternal, dolor en el pecho, indigestión, agrieras & 0 & 1 & 2 & 3 & 4 & 5 \\
\hline
\end{tabular}

0 =no molesto; 5 =problema severo / muy molesto. Adaptada de Belafsky PC, Postma GN, Koufman JA. Validity and reliability of the reflux symptom index (RSI). J Voice 2002; 16: 274-77.

Tabla 2. Puntaje de hallazgos endoscópicos de reflujo

\begin{tabular}{|l|l|}
\hline Hallazgos & Puntaje \\
\hline Pseudosulcus & 0 =ausente, 2 =presente \\
\hline Obliteración del ventrículo & 0 =no hay, 2 =parcial, 3 =completa \\
\hline Eritema / hiperemia & 0 =no hay, 2 =aritenoides, 3 =difuso \\
\hline Edema de pliegue vocal & 0 =no hay, 2 =leve, 3 =severo, 4 =polipoideo \\
\hline Edema laríngeo difuso & 0 =no hay, 2 =leve, 3 =severo, 4 =obstructivo \\
\hline Hipertrofia de la comisura posterior & 0 =no hay, 2 =leve, 3 =severo, 4 =obstructivo \\
\hline Granuloma / tejido de granulación & 0 =ausente, 2 =presente \\
\hline Moco espeso endolaríngeo & 0 =ausente, 2 =presente \\
\hline
\end{tabular}

Adaptada de Belafsky PC, Postma GN, Koufman JA. The validity and reliability of the reflux finding score (RFS). Laryngoscope 2001; 111: 1313-7. 
de este nuevo cuestionario es que es capaz de discriminar entre los pacientes que presentan RFL de aquellos que no lo presentan.

\section{Detección de pepsina}

Según lo comentado anteriormente, la pepsina juega un rol relevante en la fisiopatología de los desórdenes relacionados con reflujo. Es así que su detección se ha considerado como un marcador diagnóstico en el $\mathrm{RFL}^{30} \mathrm{y}$ es posible pesquisarla en secreciones de vía aérea cuando el reflujo gástrico ha ocurrido por un período de tiempo considerable. En 2010, Wang y cols $^{31}$ correlacionaron concentraciones de pepsina en secreción oral $>0,108 \mathrm{ng} / \mathrm{ml}$ detectada por ELISA con RSI $>10$, RFS $>8$ y en grupos de pacientes con síndrome de apnea obstructiva del sueño moderada a severa. Ellos concluyeron que existen diferencias significativas en los niveles de pepsina en secreción oral de pacientes con RFL y pacientes sanos $(p=0,000)$. La concentración de pepsina promedio en el grupo de RFL fue de $199 \mathrm{ng} / \mathrm{ml}$, consistente con lo reportado por Knight y cols ${ }^{31}$ y muy por debajo de la concentración de pepsina en jugo gástrico que es de $269 \mathrm{ng} / \mathrm{ml}$.

Hace algunos años apareció en el mercado un dispositivo de detección de pepsina en saliva (PepTest $^{\circledR}$ - RT Biomed, www.rdbiomed.com) que se encuentra disponible en los últimos meses en nuestro país. Es un método simple, de bajo costo, no invasivo, que permite detectar pepsina en saliva a través de 2 anticuerpos monoclonales contra pepsina-3, uno para detectar y otro para capturar la pepsina si está presente en la muestra. Este test tiene la capacidad de detectar pepsina en saliva en torno a una concentración de $16 \mathrm{ng} / \mathrm{ml}$ hacia arriba y se puede realizar una interpretación semicuantitativa de su concentración según la intensidad de la señal de positividad respecto del control. No existen muchos estudios aún que demuestren su real utilidad, pero el rendimiento del test según Saritas y cols ${ }^{33}$ arrojan una Sensibilidad $=50 \%$, Especificidad $=92 \%$, VPP $=85 \%$ y VPN $=68 \%$ en el grupo de pacientes con ERGE definido por esofagitis y pH-metría alterada. Este fue el primer estudio con un " $n$ " considerable, prospectivo y ciego que menciona las características de este test tanto in vitro como en pacientes con RGE. Uno de los beneficios de este test sería la posibilidad de prescindir de una endoscopia digestiva alta y/o pH-metría en los casos de PepTest positivo.

\section{CONCLUSIÓN}

El RFL es una condición tremendamente prevalente que, pese al notable aumento de las investigaciones en la última década, sigue siendo motivo de discusión, tanto en su fisiopatología como en los criterios diagnósticos. La tecnología diagnóstica ha mejorado de forma considerable en estos años pero aún su diagnóstico sigue siendo motivo de controversias. Es importante mantenerse al día en los adelantos en el estudio de su fisiopatología y en los nuevos métodos diagnósticos, así como realizar una evaluación crítica de ellos, con tal de irlos agregando paulatinamente a la práctica clínica diaria.

\section{BIBLIOGRAFÍA}

1. Groome M. Prevalence of Laryngopharyngeal Reflux in a Population With Gastroesophageal Reflux. Laryngoscope 2007; 117: 1424-8.

2. Vakil N, Van Zanten $S$, Kahrillas P, et al. The Montreal definition and Classification of GERD: a global evience-based consensus. Am J Gastroenterol 2006; 101: 1900-20.

3. $\mathrm{PoH}_{\mathrm{OH}} \mathrm{H}, \mathrm{G}$. A.-R. Upper GI tract finsdigs in patients with hearthburn in whom proton pump inhibitor treatment failed vs those not receiving antireflux treatment. Gastrointest Endosc 2010; 71: 28-34.

4. Lacy B, Weiser K, Chertoff J, et al. The diagnosis of GERD. Am J Med 2010; 123: 583-92.

5. Kollarik M, Ru F, Undem B, et al. Acid-sensitive vagal sensory pathways and cough. Pulm Pharmacol Ther 2007; 20: 402-11.

6. Galli J, Calo L, Agostino S, et al. Bile Reflux as posible risk factor in laryngopharyngeal inflammatory and neoplastic lesions. Acta Otorhinolaryngol Ital 2003; 23: 377-82.

7. Mahmoud A, Shruti P, et al. Bile Acids in Laryngopharyngeal Refluxate: Will They Enhance or Attenuate the Action of Pepsin? Laryngoscope 2013; 123: 434-9.

8. Habesoglu T, Habesoglu M, Sürmel M, et al. Histological changes of rat soft palate with expopsure to experimental laryngopharyngeal reflux. Auris Nasus Larynx 2010; 37: 730-6.

9. ERickson E, Sivasankar M. Simulated reflux decreases vocal fold epitelial barrier resistance. Laryngoscope 2010; 120: 1569-75. 
10. Johnston N, Knight J, Dettmar PW, et al. Pepsin and carbonic anhydrase isoenzyme III as diagnostic markers for laryngopharyngeal reflux disease. Laryngoscope 2004; 114: 2129-34.

11. Johnston N, Dettmar P, Lively M, et al. Effect of pepsin on laryngeal stress protein (Sep70, Sep53, and Hsp70) response: role in laryngopharyngeal reflux disease. Ann Otol Rhinol Laryngol 2006; 115: 47-58.

12. Johnston N, Dettmar P, Lively M, et Al. Activity/Stability of Human Pepsin: Implications for Reflux Attributed Laryngeal Disease. Laryngoscope 2007; 117: 1036-9.

13. Groome M, Cotton JP, Borland M, et al. Prevalence of laryngopharyngeal reflux in a population with gastroesophageal reflux. Laryngoscope 2007; 117: 1424-8.

14. Brodsky L, CarR LL. Extraesophageal reflux in children. Curr Opin Otolaryngol Head Neck Surg 2006; 14: 387.

15. Kotby MN, Hassan 0, El-Makhzangy AM et al. Gastroesophageal reflux - laryngopharyngeal reflux disease: a critical analysis of the literature. Eur Arch Otorhinolaryngol 2010; 267(2): 171-9.

16. Scott D, Simon R. Supraesophageal reflux disease: A review of the literature. Allergy Asthma Proc 2014; 35: 104-10.

17. Park W, Hicks DM, Khandwala, et AL. Laryngophariyngeal reflux: Prospective cohort study evaluating optimal dose of proton-pump inhibitor therapy and pretherapy predictors of response. Laryngoscope 2005; 115: 1230-8.

18. VaEzI MF. Treatment with PPIs should not be preceded by $\mathrm{pH}$ monitoring in patient suspected of laryngeal reflux. Am J Gastroenterol 2006; 101: 8-10.

19. Ayazi S, Hagen JA, Zehetner J, et al. Loss of alkalization in proximal esophagus: a new diagnostic paradigm for patients with laryngopharyngeal reflux. J Gastrointest Surg 2010; 14: 1653-9.

20. Hawkshaw M, Pebdani P, Sataloff R. Reflux Laryngitis: An Update. Journal of Voice 2013; 27 (4): 486-94.

21. Sun G, Muddana S, Slaugther JC, et al. A new pH catheter for laryngopharyngeal reflux: normal values. Laryngoscope 2009; 119: 1639-43.

22. Wiener G, Tsukashima R, Kelly C, et al. Oropharyngeal $\mathrm{pH}$ monitoring for the detection of liquid and aerosolized supraesophageal gastric reflux. Journal of Voice 2009; 101: 498-504.

23. Sato K, Umeno H, Chitose S, et al. Tetra probe, 24hour $\mathrm{pH}$ monitoring for laryngopharingeal reflux;a technique for simultaneous study of hypopharynx, esophagus and stomach. J Laryngol Otol 2009; 123 (s31): 117-22.

24. Mousa H, Rosen R, Woodley F, et al. Monitoreo esofágico por impedanciometría para el reflujo gastroesofágico. Acta Gastroenterológica Latinoam 2011; 41(2): 146-61.

25. Lee BE, KIm GH, Ruy D, et AL. Combined dual channel impedance/pH-metry in patients with suspected Laryngopharyngeal reflux. J Neurogastroenterol Motil 2010; 16: 157-65.

26. Loots CM, Benninga MA, Davidson GP, Omari TI. Addition of pH-impedance monitoring (ph-MII) to standard pH monitoring increases the yield of symptom association analysis in infants and children with gastroesophageal reflux. J Pediatr 2009; 154: 248-52.

27. Belafsky PC, Postma GN, Koufman JA. The validity and reliability of the reflux symptom index (RSI). Journal of Voice 2002; 16: 274-7.

28. Belafsky PC, Postma GN, Koufman JA. The validity and reliability of the reflux finding score (RFS). Laryngoscope 2001; 111: 1313-7.

29. Andersson 0, Ryden A, Ruth M, Moller RY, Finizia C. Development and validation of a laryngopharyngeal reflux questionnaire, the Pharyngeal Reflux Symptoms Questionnaire. Scand J Gastroenterol 2010; 45: 14759.

30. Samuels TL, Johnston N. Pepsin as a marker of extraesophageal reflux. Ann Otol Rhinol Laryngol 2010; 119: 203-8.

31. Wang L, LiU X, LiU y, et al. Correlation of pepsinmeasured laryngopharyngeal reflux disease with symptoms and signs. Otolaryngol Head Neck Surg 2010; 143: 765-71.

32. Knight J, Lively MO, Johnston N, et al. Sensitive pepsin immunoassay for detection of laryngopharyngeal reflux. Laryngoscope 2005; 115: 1473-8.

33. Saritas E, Hong S, Strugala V, et al. Rapid Salivary Pepsin Test: Blinded Assesment of Test Performance in Gastroesophageal Reflux Disease. Laryngoscope 2012; 122: 1312-6.

Dirección: Christian Olavarría Leiva

Servicio de Otorrinolaringología, Hospital Clínico Universidad de Chile.

Santos Dumont 999, Independencia. Santiago

E mail: christian.olavarria@gmail.com 